NASA Contractor Report 178123

ICASE REPORT NO. $86-33$

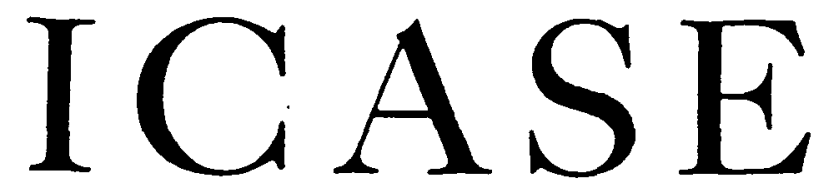

NASA-CR-178123

19860020949

A MINIMUM ENTROPY PRINCIPLE IN

THE GAS DYNAMICS EQUATIONS

Eitan Tadmor

Contract Nos. NAS1-17070, NAS1-18107

May 1986

INSTITLTE FOR CONPUTER APPLICATIONS IN SCIENCE AND ENGINEERING NASA Langley Research Center, Hampton, Virginia 23665

Operated by the Universities Space Research Association

\section{N/SA}

National Aeronautics and Space Administration Langley Research Center Hampton. Virginia 23665
LIRARY RAPM

$$
19096960
$$

LANGLEY RESEARCH CENTER LIBRARY, NASA

HAA:PTON, VIRGIIIIA 


\title{
A MINIMUM ENTROPY PRINCIPLE IN THE GAS DYNAMICS EQUATIONS
}

\author{
Eitan Tadmor* \\ School of Mathematical Sciences, Tel-Aviv University \\ and \\ Institute for Computer Applications in Sclence and Engineering \\ Dedicated to Milton E. Rose \\ on Occasion of his 60 th Birthday
}

\begin{abstract}
Let $\mathbf{u}(\bar{x}, t)$ be a weak solution of the Euler equations, governing the inviscid polytropic gas dynamics; in addition, $u(\bar{x}, t)$ is assumed to respect the usual entropy conditions connected with the conservative Euler equations. We show that such entropy solutions of the gas dynamics equations satisfy a minimum entropy principle, namely, that the spatial minimum of their specific entropy, Ess inf $s(u(\bar{x}, t))$, is an increasing function of time. This principle equally applies to discrete approximations of the Euler equations such as the Godunov-type and Lax-Friedrichs schemes. Our derivation of this minimum principle makes use of the fact that there is a family of generalized entropy functions connected with the conservative Euler equations.
\end{abstract}

Research was supported in part by NASA Contract No. NAS1-17070 while the author was in residence at ICASE, NASA Langley Research Center, Hampton, VA 23665-5225. Additional support was provided in part by NSF Grant No. DMS8503294 and ARO Grant No. DAAG29-85-K-0190 while in residence at the University of California, Los Angeles, CA 90024.

*Bat-Sheva Foundation Fellow 


\section{INTRODUCTION}

Many phenomena in continuum mechanics are modeled by hyperbolic systems of conservation laws

$$
\frac{\partial u}{\partial t}+\sum_{k=1}^{d} \frac{\partial f^{(k)}}{\partial x_{k}}=0, \quad\left(\bar{x}=\left(x_{1}, \cdots, x_{d}\right), t\right) \in \underline{R} \times[0, \infty),
$$

where $f^{(k)} \equiv f^{(k)}(u)=\left(f_{1}^{(k)}, \cdots, f_{N}^{(k)}\right)^{T}$ are smooth nonlinear flux mappings of the N-vector of conservative variables $u \equiv u(\bar{x}, t)=\left(u_{1}, \cdots, u_{N}\right)^{T}$. Friedrichs and Lax [3] have observed that the hyperbolic nature of such models is revealed by the property of most of those systems being endowed with a generalized

Entropy Function: A smooth convex mapping $U(\mathbf{u})$ augumented with entropy flux mappings $\quad \vec{F} \equiv \vec{F}(\mathbf{u})=\left(F^{(1)}(\mathbf{u}), \cdots, F^{(d)}(\mathbf{u})\right)$, such that the following compatibility relations hold

$$
\mathrm{U}_{\mathbf{u}}^{\mathrm{T}} \mathbf{f}_{\mathbf{u}}^{(\mathrm{k})}=\mathrm{F}_{\mathbf{u}}^{(\mathrm{k}) \mathrm{T}}, \quad \mathrm{k}=1,2, \cdots, \mathrm{d} .
$$

Multiplying (1.1) by $U_{u}^{T}$ and employing (1.2), one arrives at an equivalent formulation of the compatibility relations (1.2), namely, that under the smooth regime we have on top of $(1.1)$ the additional conservation of entropy

$$
\frac{\partial U}{\partial t}+\sum_{k=1}^{d} \cdot \frac{\partial F(k)}{\partial x_{k}}=0
$$

Owing to the nonlinearity of the fluxes $f^{(k)}(u)$, solutions of (1.1) may develop singularities at a fintte time after which one must admit weak 
solutions, i.e., those derived directly from the underlying Integral conservative equations. Considering (1.1) as a strong limit of the regularized problem,

$$
\frac{\partial \mathbf{u}}{\partial t}+\sum_{k=1}^{d} \frac{\partial \mathbf{f}^{(k)}}{\partial x_{k}}=\mu \sum_{k=1}^{d} \frac{\partial^{2} \mathbf{u}}{\partial x_{k}^{2}}, \mu+0,
$$

then following Lax [9] and Krushkov [8], we postulate as an admissibility criterion for such limit solutions an entropy stability condition which manifests itself in terms of an

Entropy Inequality: We have, in the sense of distributions,

$$
\frac{\partial U}{\partial t}+\sum_{k=1}^{d} \frac{\partial F^{(k)}}{\partial x_{k}} \leqq 0
$$

Weak solutions of (1.1), which in addition satisfy the inequality (1.5) for all entropy pairs (U,F) connected with that system, are called entropy solutions. (1) Having a (weakly) nonpositive quantity on the L.H.S. of (1.5) is thus a consequence of viewing these entropy solutions as limits of vanishing dissipativity mechanisms. In particular, the inequality (1.5) implies that the total entropy in the domain decreases in time (we assume entropy outflux through the boundaries)

$$
\frac{d}{d t} \int_{\bar{x}} U(\mathbf{u}(\bar{x}, t)) d \bar{x} \leq 0
$$

\footnotetext{
(1) Krushkov [8, p. 241] has termed such solutions simply as generalized solutions.
} 
In this paper, we consider entropy solutions,

$$
\mathbf{u}=(\rho, \mathbf{m}, E)^{\mathrm{T}}
$$

of the Euler equations. These equations govern the Inviscid polytropic gas dynamics, asserting the conservation of the density $\rho$, the momentum $m=\left(m_{1}, m_{2}, m_{3}\right)^{T}$, and the energy E. Let $q \equiv \frac{m}{p}$ denote the velocity field of such motion. Then, expressed in terms of the pressure, p,

$$
p=(\gamma-1) \cdot\left[E-1 / 2 \cdot p|q|^{2}\right], \quad \gamma=\text { adiabatic exponent }
$$

the corresponding fluxes in this case are given by (2)

$$
\mathbf{f}^{(k)}=\left(m_{k}, q_{k} \cdot \mathbf{m}+p \cdot e^{(k)}, q_{k}(E+p)\right)^{T}, \quad k=1,2,3
$$

The main result of this paper asserts that entropy solutions of Euler equations satisfy the following

Mintmum Principle: Let $u \equiv u(\bar{x}, t)$ be an entropy solution of the gas dynamics equations (1.7) and let

$$
s(\bar{x}, t) \equiv s(u(\bar{x}, t))-\ln \left(p p^{-\gamma}\right)
$$

${ }^{(2)}$ With $e^{(k)}$ denoting the unit Cartesian vectors $e^{(k)}=\delta_{k j}$. 
denote the specific entropy of such solution. Then the following estimate holds

$$
\begin{aligned}
& \text { Ess inf } s(\bar{x}, t) \geqq \quad \text { Ess inf } s(\bar{x}, t=0) . \\
& |\bar{x}| \leqq R
\end{aligned}
$$

Here $q_{\max }$ stands for the maximal speed $|\mathbf{q}|$ in the domain.

The proof of this assertion is provided in Section 3 below. Prior to that we elaborate in Section 2 on the entropy inequality connected with the gas dynamics equations. In particular, Harten [5] has shown that there exists a whole family of entropy pairs associated with these equations, a fact which is essential in our derivation of the minimum principle.

As an immediate consequence of the minimum princlple, we conclude that Ess inf $S(\bar{x}, t)$ is an increasing function of $t$ for every entropy solution of (1.7). The following argument sheds additional light on this conclusion in the case of a plecewise-smooth flow. To this end, an arbitrary particle currently located at $(\bar{x}, t)$ is traced backwards in time into its initial position at $t=0$. Since the specific entropy of such particle remains constant along the particle path--except for its decrease when crossing backwards shock waves, it follows that its value $s(\bar{x}, t)$ is greater or equal than that of the initial spatial minimum Ess inf $s(\bar{x}, t=0)$, as asserted. In contrast to the above 'Lagrangian' argument, the derivation of the minimum principle outlined below, is purely an "Eulerian' one. It enables us to relax the regularity assumption on the flow, and--since we do not follow the characteristics, it equally applies to discrete approximations of the Euler equations. 
In Section 4 we consider approximate solutions of the Euler equations, $w\left(\bar{x}_{v}, t\right)$, wh1ch respect the entropy decrease estimate (1.6),

$$
\sum_{v} U\left(w\left(\bar{x}_{v}, t+\Delta t\right)\right) \Delta \bar{x}_{v} \leq \sum_{v} U\left(w\left(\bar{x}_{v}, t\right)\right) \Delta \bar{x}_{v}
$$

We note that such approximate solutions are obtained by entropy stable schemes satisfying the cell entropy inequality

$$
U\left(w\left(\bar{x}_{v}, t+\Delta t\right)\right) \leq U\left(w\left(\bar{x}_{v}, t\right)\right)+\sum_{k=1}^{d} \frac{1}{\Delta \bar{x}_{v}}\left[F_{v+1 / 2}^{(k)}-F_{v-1 / 2}^{(k)}\right],
$$

e.g., the Godunov-type and Lax-Friedrichs schemes [6]. We have

Minimum Principle: Let $w\left(\bar{x}_{v}, t\right)$ be an approximate solution of the gas dynamics equations $(1.8)$ and let

$$
\left.s\left(\bar{x}_{v}, t\right) \equiv s\left(\nabla_{v}, t\right)\right)=\ln \left(p p^{-\gamma}\right)
$$

denote the specific entropy of such solution. Assume that its total entropy decreases in time, $(1,10)$. Then the following estimate holds

$$
S(\bar{x}, t+\Delta t) \geqq \underset{v}{\operatorname{Min}}\left[S\left(\bar{x}_{v}, t\right)\right]
$$

In the case of entropy stable schemes, (1.11), a more precise estimate is obtained which takes into account the support of the schemes' stencil.

The inequality (1.13) leads to an apriori pointwise estimate on the approximate solution $w(\bar{x}, t)$. Such pointwise estimates play an essential role 
with regard to question of the convergence of entropy stable schemes. In particular, DiPerna [2, Section 7] has recently shown that in certain cases, such (two-sided) estimates are sufficient in order to guarantee the convergence of such schemes.

\section{GENERALIZED ENTROPY FUNCTIONS OF THE EULER EQUATIONS}

We consider the Euler equations for polytropic gas

$$
\frac{\partial}{\partial t}\left[\begin{array}{l}
\rho \\
m \\
E
\end{array}\right]+\sum_{k=1}^{3} \frac{\partial}{\partial x_{k}}\left[\begin{array}{c}
m_{k} \\
q_{k} m+p e^{(k)} \\
q_{k}(E+\rho)
\end{array}\right]=0
$$

It is well-known, e.g., [1], that for all smooth solutions of (2.1) the specific entropy $(3)$

$$
s(\bar{x}, t)=\ln \left(p^{-\gamma}\right),
$$

remains constant along streamlines, 1.e.,

$$
\frac{D S}{D t}=\frac{\partial S}{\partial t}+\sum_{k=1}^{3} q_{k} \frac{\partial S}{\partial x_{k}}=0
$$

Let $h(S)$ be an arbitrary smooth function of $S$. Multiplying (2.2a) by $p h^{-}(S)$-prime denoting s-differentiation, we find

(3) After normalization, taking the specific heat constant to be $c_{v}=1$. 


$$
\rho \frac{\partial h(S)}{\partial t}+\sum_{k=1}^{3} m_{k} \frac{\partial h(S)}{\partial x_{k}}=0
$$

Adding this to the continulty equation which is premultiplied by $h(S)$,

$$
\frac{\partial \rho}{\partial t} h(s)+\sum_{k=1}^{3} \frac{\partial m_{k}}{\partial x_{k}} h(s)=0
$$

we obtain after changing sign, a conservative entropy equation 11ke (1.3) which reads [5]

$$
\frac{\partial}{\partial t}[-\rho h(s)]+\sum_{k=1}^{3} \frac{\partial}{\partial x_{k}}\left[-m_{k} h(s)\right]=0 .
$$

In order to comply with the further requirement of being a generalized entropy function, $U(u)=-\rho h(S)$ has to be a convex function of the conservative variables $\mathbf{u}=(\rho, \mathbf{m}, E)^{\mathrm{T}}$. A straightforward computation carried out by Harten [5, Section 2] in the two-dimensional case shows that the Hessian $U_{u u}$ is positive definite if and only if

$$
\rho\left[h^{-}(S)-\gamma \cdot h^{-\prime}(S)\right]>0
$$

Excluding negative densities we may summarize that there exists a family of (generalized) entropy pairs (U,F) associated with Euler equations (2.1),

$$
U(u)=-\rho h(s), \quad F^{(k)}(u)=-m_{k} h(s) \quad k=1,2,3,
$$

generated by the smooth increasing functions $h(S)$ which satisfy 


$$
h^{\prime}(s)-\gamma \cdot h^{\prime \prime}(s)>0
$$

\section{A MINIMUM ENTROPY PRINCIPLE}

Let $u=(\rho, E, E)^{T}$ be an entropy solution of the gas dynamics equations (2.1). Such a solution is characterized by the entropy inequality (1.7)

$$
\frac{\partial U(\mathbf{u})}{\partial t}+\sum_{k=1}^{3} \frac{\partial F^{(k)}(\mathbf{u})}{\partial x_{k}} \leqq 0
$$

which holds for all entropy pairs (U, $\vec{F})$ connected with the equations.

To derive a minimum principle, we shall make use of an argument due to Lax $[9$, Section 3]. We begin with

Lemma 3.1: Let $u$ be an entropy solution of the gas dynamics equations (2.1). Then for all nonpositive smooth increasing functions $h(S)$ satisfying $(3.2 \mathrm{~b})$, we have

$$
\int_{|\bar{x}| \leqq R} \rho(\bar{x}, t) \cdot h(s(\bar{x}, t)) d \bar{x} \geqq \int_{|\bar{x}| \leqq R+t \cdot q_{\max }} \rho(\bar{x}, 0) \cdot h(s(\bar{x}, 0)) d \bar{x}
$$

Here $q_{\max }$ denotes the maximal speed $|q|$ in the domain.

Proof: As in [10, Theorem 4.1] we integrate the entropy inequality (3.2a) over the truncated cone $C=\left\{|\bar{x}| \leqq R+(t-\tau) \cdot q_{\max } \mid 0 \leqq \tau \leqq t\right\}$; if we let $\left(n_{0}, \bar{n}\right)$ denote the unit outward normal, then by Green's theorem 


$$
\int_{\partial C} \rho h(s) \cdot\left[n_{0}+\sum_{k=1}^{3} q_{k} n_{k}\right] \partial \bar{x} \geqq 0 .
$$

The integrals over the top and bottom surfaces give us the difference between the left and right-hand sides in (3.3) and by (3.4) this difference is bounded from below by

$$
-\int_{\operatorname{mantle}} \rho h(s) \cdot\left[n_{0}+\sum_{k=1}^{d} q_{k} n_{k}\right] \partial \bar{x} .
$$

The result follows upon showing that the last quantity is nonnegative. Indeed, since by assumption $-\rho h(S) \geqq 0$, this is the same thing as

on the mantle we have

$$
\mathrm{n}_{0}+\sum_{k=1}^{3} \mathrm{q}_{k} \mathrm{n}_{\mathrm{k}} \geq 0
$$

$$
\left(n_{0}, \bar{n}\right)=\frac{1}{\sqrt{1+q_{\max }^{2}}}\left(q_{\max }, \frac{\bar{x}}{|\bar{x}|}\right),
$$

and hence

$n_{0}+\sum_{k=1}^{3} q_{k} n_{k}=\frac{1}{\sqrt{1+q_{\max }^{2}}}\left(q_{\max }+\sum_{k=1}^{3} \frac{q_{k} x_{k}}{|\bar{x}|}\right) \geq \frac{1}{\sqrt{1+q_{\max }^{2}}}\left(q_{\max }-\sum_{k=1}^{3} \frac{\left|q_{k}\right|^{2}}{|q|}\right) \geq 0$ as asserted.

The discussion in Lemma 3.1 was restricted to smooth function $h(S)$; by passing to the limit, its conclusion (3.3) follows for any nonpositive nondecreasing function $h(S)$ satisfying (3.2b), whether smooth or not. 
To derive the minimum entropy principle, we now make a special choice of such function, $h(s)$, given by

$$
\begin{aligned}
& h(s)=\operatorname{Min}\left[s-s_{0}, 0\right], \quad s_{0}=\text { Ess inf } s(\bar{x}, 0) \text {. } \\
& |\bar{x}| \leqq R+t \cdot q_{\max }
\end{aligned}
$$

The nonpositive function $h(S)$. Is a nondecreasing concave, one, hence admissible by (3.2b), and consequently (3.3) applies

$$
\int_{|\bar{x}| \leq R} \rho(\bar{x}, t) \cdot \operatorname{Min}\left[s(\bar{x}, t)-s_{0}, 0\right] d \bar{x} \geq
$$

Now, by the choice of $s_{0}$, the integral on the right of (3.6) vanishes since $\operatorname{Min}\left[S(\bar{x}, 0)-S_{0}, 0\right]$ does. The inequality (3.6) then tells us that the integral on the left is also nonnegative. But since the integrand on the left is by definition nonpositive, this can be the case provided this integrand vanishes almost everywhere; that is, we have for almost all $\bar{x},|\bar{x}| \leqq R$

$$
s(\bar{x}, t) \geqq S_{0}=\begin{aligned}
& \text { Ess inf } \\
& |\bar{x}| \leqq R+t \cdot q_{\max }
\end{aligned}
$$

and (1.9) follows.

The minimum entropy principle was deduced from the entropy inequality (3.2), which in turn was postulated based on the formal regularization introduced in (1.4). In general, other regularizations equally apply; in 
particular, Euler equations are usually sought as the vanishing viscosity limit of the Navier-Stokes equations (here we take for simplicity the onedimensional case) (4)

$$
\frac{\partial}{\partial t}\left[\begin{array}{l}
\rho \\
m \\
E
\end{array}\right]+\frac{\partial}{\partial x}\left[\begin{array}{c}
m \\
q m+p \\
q(E+p)
\end{array}\right]=\mu \frac{\partial}{\partial x}\left[\begin{array}{c}
0 \\
\frac{\partial q}{\partial x} \\
q \frac{\partial q}{\partial x}
\end{array}\right], \quad \mu+0
$$

Do the (generalized) entropy inequalities (3.2) remain valid on the basis of such limit? To answer this question we first note that if $U(u)$ is any entropy function, then thanks to its convexity the mapping $\mathbf{u}+\mathbf{v} \equiv U_{\mathbf{u}}$ is one-to-one, and hence one can make the change of variables $\mathbf{u}=\mathbf{u}(\mathbf{v})$. Harten [5] has shown that such change of variables by each member of the family of entropy functions (2.4) puts the viscosity terms on the right of (3.7) into a negative semidefinite form. This makes apparent the dissipative effect of these viscosity terms. Indeed, if $T=c_{v} \cdot E-1 / 2 \cdot|q|^{2}$ denotes the absolute temperature, then direct manipulation of (3.7) yields, e.g., [1, Section 63], [12, Section 6.10],

$$
\frac{\partial}{\partial t}[\rho h(s)]+\frac{\partial}{\partial x}[\operatorname{mh}(S)]=\mu \cdot h(s) \frac{q_{x}^{2}}{T},
$$

from which we recover the entropy inequality (3.2a) for all smooth increasing functions $h(S)$. We note that the convexity condition was not assumed in this

(4) With in combining the two viscosity coefficients in the general NavierStokes equations. 
case. The merit of using the convexity condition, however, is that it enables us to deal with more general artificial viscosity terms, other than those appearing in the Navier-Stokes equations. Such artificial viscosity terms are frequently encountered in finite-difference approximations to the Euler equations; a specific example of this kind is studied in the next section.

Finally we would like to remark on the previously mentioned Navier-Stokes equations. Our discussion above took into account only the viscosity contribution, neglecting heat conduction. Hughes, et al., [7] have shown that when the heat flux is also added, compare (3.7),

$$
\frac{\partial}{\partial t}\left[\begin{array}{l}
\rho \\
m \\
E
\end{array}\right]+\frac{\partial}{\partial x}\left[\begin{array}{c}
m \\
q m+p \\
q(E+p)
\end{array}\right]=\mu \frac{\partial}{\partial x}\left[\begin{array}{c}
0 \\
\frac{\partial q}{\partial x} \\
q \frac{\partial q}{\partial x}
\end{array}\right]+\kappa \frac{\partial}{\partial x}\left[\begin{array}{l}
0 \\
0 \\
\frac{\partial T}{\partial x}
\end{array}\right]
$$

with $K$ denoting the heat conductivity constant, then only the 'physical' entropy, $U(u)=-o S$ survives as the one which puts the additional heat flux into a symmetric negative-definite form. We would like to note in this connection the difference limit behavior of the Navier-Stokes flows depending on the viscosity and heat conductivity; Gilbarg [4] has shown that as $k \rightarrow 0$ keeping $\mu$ fixed, we are led to a continuous thermally nonconducting shock layer, whereas for $\mu+0$ with $k$ fixed the convergence is to a (generally) discontinuous nonviscous shock layer. Consequently, the viscosity rather than the heat flux should play the major rule in an appropriate regularization model for the Euler equations. 


\section{DISCRETE APPROXIMATIONS OF THE EULER EQUATIONS}

In this section we consider approximate solutions of the Euler equations, $w\left(x_{v}, t\right)$, whose total entropy decreases in time, compare $(1.10)$

$$
\sum_{v} U\left(w\left(\bar{x}_{v}, t+\Delta t\right)\right) \Delta \bar{x}_{v} \leq \sum_{v} U\left(v\left(\bar{x}_{v}, t\right)\right) \Delta \bar{x}_{v}
$$

Estimate (4.1) holds for all entropy functions $U=-\rho h(s)$ in (2.4). By passing to the limit, this applies to our previous choice of the function $h(s)$ in (3.5)

$$
h(s)=\operatorname{Min}\left[s-s_{0}, 0\right]
$$

this time with a constant $S_{0}$ which is taken to be

$$
S_{0}=\operatorname{Min}_{v} S\left(w\left(\bar{x}_{v}, t\right)\right)
$$

By our choice of $S_{0}$, we have $U\left(w\left(\bar{x}_{v}, t\right)\right)=0$. The inequality (4.1) tells us that the left-hand side is therefore, nonnegative; consequently

$$
s(\bar{x}, t+\Delta t)-S_{0} \geq h(S(x, t+\Delta t)) \geq 0
$$

and (1.13) follows.

Approximate solutions which fulfill the required estimate (4.1) can be obtained by entropy stable schemes satisfying the cell entropy inequality (1.11)

$$
U\left(w\left(\bar{x}_{v}, t+\Delta t\right)\right) \leq U\left(v\left(\bar{x}_{v}, t\right)\right)+\sum_{k=1}^{d} \frac{1}{\Delta \bar{x}_{v}}\left[F_{v+1 / 2}^{(k)}-F_{v-1 / 2}^{(k)}\right]
$$


Examples of such entropy stable schemes Include the Godunov-type and LaxFriedrichs schemes, e.g., [6]. A more precise minimum principle follows in these cases, taking into account the support of the schemes stencil. In particular, the (one-dimensional) Godunov scheme results from averaging of two nelghboring Rlemann problems [6], each of which satisfies (1.9). Consequently we have the

Minimum Principle (of the Godunov scheme): Let $w\left(x_{v}, t\right)$ the Godunov
approximate solution to the Euler equations (2.1). Assume that the
appropriate CFL condition is met. Then the following estimate holds

$$
S\left(w\left(x_{v}, t+\Delta t\right)\right) \geq \operatorname{Min}_{v-1 \leq j \leq v+1} S\left(w\left(x_{j}, t\right)\right)
$$

Since the Lax-Friedrichs scheme coincides with a staggered Godunov's solver, the same conclusion, (4.4), holds. Another way to see this is outlined below; it makes no reference to Riemann's solution and can be generalized to the multidimensional problem.

To this end, we approximate the (for simplicity--one-dimensional) Euler equations with the Lax-Friedrichs scheme

$$
\begin{aligned}
w\left(x_{v}, t+\Delta t\right)=\frac{1}{2} & {\left[w\left(x_{v+1}, t\right)+w\left(x_{v-1}, t\right)\right] } \\
& \left.-\frac{\lambda}{2}\left[f\left(w\left(x_{v+1}, t\right)\right)-f\left(v_{v-1}, t\right)\right)\right], \quad \lambda \equiv \frac{\Delta t}{\Delta x} .
\end{aligned}
$$

We remark that the Lax-Friedrichs scheme can be derived from center differencing of the regularization model (1.4) $\Delta x^{\bullet}$ Lax has shown [9, Theorem 
1.2] that if $\lambda \equiv \frac{\Delta t}{\Delta x}$ is sufficiently small, then solutions of this difference scheme satisfy the following cell entropy inequality

$$
\begin{aligned}
U\left(v\left(x_{v}, t+\Delta t\right)\right) \leqq & \frac{\left.U\left(w\left(x_{v+1}, t\right)\right)+U\left(v_{v-1}, t\right)\right)}{2} \\
& \left.\left.-\frac{\lambda}{2} \cdot\left[F\left(w_{v+1}, t\right)\right)-F\left(x_{v-1}, t\right)\right)\right]
\end{aligned}
$$

for all entropy pairs $(U, F)=(-p h(S),-m h(S))$. in $(2.4)$. by passing to the limit, this applies to our previous choice of the function $h(s)$ in $(3.5)$

$$
h(s)=\operatorname{Min}\left[s-s_{0}, 0\right]
$$

this time, with a contant $S_{0}$ which is taken to be

$$
s_{0}=\operatorname{Min}\left[s\left(x_{v+1}, t\right), s\left(x_{v-1}, t\right)\right]
$$

The inequality $(4.6)$ now reads

$$
\begin{aligned}
\rho\left(x_{v}, t+\Delta t\right) \cdot h\left(S\left(x_{v}, t+\Delta t\right)\right) \geqq & {\left[\frac{1+\lambda q\left(x_{v-1}, t\right)}{2} \rho\left(x_{v-1}, t\right) \cdot h\left(S\left(x_{v-1}, t\right)\right)\right.} \\
& \left.+\frac{1-\lambda q\left(x_{v+1}, t\right)}{2} \rho\left(x_{v+1}, t\right) \cdot h\left(s\left(x_{v+1}, t\right)\right)\right] .
\end{aligned}
$$

By our choice of the funtion $h(S)$ in (4.7), we have $h\left(S\left(x_{v \pm 1}, t\right)\right)=0$. The Inequality (4.8) tells us that the left-hand side is therefore nonnegative; consequently 


$$
0 \leq h\left(s\left(x_{v}, t+\Delta t\right)\right) \leq s(x, t+\Delta t)-S_{0}
$$

and the following minimum principle follows

$$
s\left(w\left(x_{v}, t+\Delta t\right)\right) \geq \operatorname{Min} S\left(w\left(x_{v \pm 1}, t\right)\right) .
$$




\section{REFBRENCES}

[1] R. Courant and K. O. Friedrichs, Supersonic Flow and Shock Waves, Intersclence, New York, 1948.

[2] R. J. DiPerna, "Convergence of approximate solutions to conservation laws," Arch. Rational Mech. Anal., Vol. 82 (1983), pp. 27-70.

[3] K. 0. Friedrichs and P. D. Lax, "Systems of conservation laws with a convex extension," Proc. Nat. Acad. Sc1. U.S.A., Vol. 68 (1971), pp. 1686-1688.

[4] D. Gilbarg, "The existence and limit behavior of the one-dimensional shock layer," Amer. J. Math., Vol. 73 (1951), pp. 256-274.

[5] A. Harten, "On the symmetric form of systems of conservation laws with entropy," J. Comput. Phys., Vol. 49 (1983), pp. 151-164.

[6] A. Harten, P. D. Lax, and B. Van Leer, "On upstream differencing and Godunov-type schemes for hyperbolic conservation laws," SIAM Rev., Vol. 25 (1983), pp. 35-61.

17] T. J. R. Hughes, L. P. Franca, and M. Mallet, "Symetric forms of the compressible Euler and Navier-Stokes equations and the second law of thermodynamics," Comput. Methods Appl. Mech. Engrg., to appear. 


$$
-18-
$$

[8] S. N. Krushkov, "First-order quasilinear equations in several Independent variables," Math. USSR-Sb., Vol. 10 (1970), pp. 217-243.

[9] P. D. Lax, "Shock waves and entropy" in Contributions to Nonlinear Functional Analysis (E. H. Zarantonello, ed.), pp. 603-634, 1971.

[10] E. Tadmor, "Skew-selfadjoint form for systems of conservations laws," J. Math. Anal. Appl., Vol. 703 (1984), pp. 428-442.

[11] E. Tadmor, "The numerical viscosity of entropy stable schemes for systems of conservation laws. I.," NASA Langley Research Center, ICASE Report 85-51, NASA CR-178021, 1985.

[12] G. Whitham, Linear and Nonlinear Waves, Wiley-Interscience, 1974. 
Standard Bibliographic Page

\begin{tabular}{|c|c|c|}
\hline $\begin{array}{l}\text { 1. Report No. NASA CR-178123 } \\
\text { ICASE Report No. } 86-33\end{array}$ & ient Accession No. & 3. Recipient's Catalog No. \\
\hline \multirow{2}{*}{\multicolumn{2}{|c|}{$\begin{array}{l}\text { 4. Title and Subtitle } \\
\text { A MINIMUM ENTROPY PRINCIPLE IN } \\
\text { THE GAS DYNAMICS EQUATIONS }\end{array}$}} & $\begin{array}{r}\text { 5. Report Date } \\
\text { May } 1986\end{array}$ \\
\hline & & 6. Performing Organization Code \\
\hline \multicolumn{2}{|l|}{$\begin{array}{l}\text { 7. Author(s) } \\
\text { Eitan Tadmor }\end{array}$} & $\begin{array}{l}\text { 8. Performing Organization Report No. } \\
86-33\end{array}$ \\
\hline \multicolumn{2}{|c|}{$\begin{array}{l}\text { 9. Performing Organization Name and Addrass cations in Science } \\
\text { and Engineering } \\
\text { Mail Stop 132C, NASA Langley Research Center } \\
\text { Hampton, VA 23665-5225 }\end{array}$} & \begin{tabular}{|l|} 
10. Work Unit No. \\
11. Contract 9 offant No.
\end{tabular} \\
\hline \multirow{2}{*}{\multicolumn{2}{|c|}{$\begin{array}{l}\text { 12. Sponsoring Agency Name and Address } \\
\text { National Aeronautics and Space Administration } \\
\text { Washington, D.C. } 20546\end{array}$}} & $\begin{array}{l}\text { 13. Type of Report and Period Covered } \\
\text { Contractor Report }\end{array}$ \\
\hline & & $\begin{array}{l}\text { 14. Sponsoring Agency Code } \\
505-31-83-01\end{array}$ \\
\hline \multicolumn{3}{|l|}{$\begin{array}{l}\text { Langley Technical Monitor: } \\
\text { J. C. South } \\
\text { Final Report }\end{array}$} \\
\hline \multicolumn{3}{|c|}{$\begin{array}{l}\text { 16. Abstract } \\
\text { Let } u(\bar{x}, t) \text { be a weak solution of the Euler equations, governing the } \\
\text { inviscid polytropic gas dynamics; in addition, } u(\bar{x}, t) \text { is assumed to } \\
\text { respect the usual entropy conditions connected with the conservative Euler } \\
\text { equations. We show that such entropy solutions of the gas dynamics equations } \\
\text { satisfy a minimum entropy principle, namely, that the spatial minimum of their } \\
\text { specific entropy, Ess inf } s(u(x, t)) \text {, is an increasing function of time. } \\
\text { This principle equally applies to discrete approximations of the Euler } \\
\text { equations such as the Godunov-type and Lax-Friedrichs schemes. Our derivation } \\
\text { of this minimum principle makes use of the fact that there is a family of } \\
\text { generalized entropy functions connected with the conservative Euler equations. }\end{array}$} \\
\hline \multicolumn{3}{|c|}{\begin{tabular}{l|c} 
17. Key Words (Suggested by Authors(s)) & 18. Distribution Statement \\
$\begin{array}{l}\text { Euler equations, finite differences, } \\
\text { Godunov scheme, Lax-Friedrichs scheme }\end{array}$ & 64 - Numerical Analysis \\
& Unclassified - unlimited
\end{tabular}} \\
\hline $\begin{array}{l}\text { 19. Security Classif.(of this report) } \\
\text { Unclassified }\end{array}$ & $\begin{array}{l}\text { Classif.(of this page) } \\
\text { classified. }\end{array}$ & \begin{tabular}{|c|c|} 
21. No. of Pages & 22. Price \\
20 & $\mathrm{~A} 02$
\end{tabular} \\
\hline
\end{tabular}




\section{End of Document}

